\title{
Tidal Disruption Events and stellar-mass black holes in OGLE and Gaia surveys
}

\author{
Warsaw University Astronomical Observatory \\ Al. Ujazdowskie 4, Warszawa 00-478, Poland \\ email: 1w@astrouw.edu.pl
}

Łukasz Wyrzykowski, A. Hamanowicz and K. A. Rybicki

\begin{abstract}
Tidal Disruption Events (TDE) allow to probe the super massive black holes (SMBH) in the cores of galaxies and could be a source of black hole mass growth. We present the search for candidates for TDEs conducted within OGLE and Gaia surveys. Our preliminary results indicate that TDEs can occur in cores of galaxies exhibiting different levels of activity, from quiescent, through weak-AGNs to highly active QSOs. We also present how Gaia can help study the mass distribution of Milky Way single black holes via microlensing.
\end{abstract}

Keywords. super massive black holes, tidal disruption events, OGLE, Gaia

\section{Introduction}

Tidal Disruption Events (TDE) offer an opportunity to detect and study Super Massive Black Holes (SMBH) at lower-end of their mass distribution. However, the number statistics are still too low what makes it difficult to employ TDEs as a useful tool. Moreover, there is still no consensus about the nature of some nuclear flares being found both in real-time and in archives, e.g., changing-look QSOs/AGNs (Shappee et al. 2014, LaMassa et al. 2015, MacLeod et al. 2016, Ruan et al. 2016) or slow-blue hypervariables (Lawrence et al. 2016). New, well observed and studied examples are necessary and here we present our search for nuclear flares from OGLE and Gaia surveys. Additionally, we describe how Gaia will help measure the mass distribution of stellar-mass black holes in the Milky Way via gravitational microlensing.

\section{$O G L E$}

OGLE $\dagger$ is a Polish large-scale photometric monitoring survey, operating since 1992, using a dedicated $1.3 \mathrm{~m}$ telescope in Las Campanas Observatory, Chile. Since 2010 (OGLE-IV) it utilises the 32-chip CCD mosaic camera with field-of-view of 1.4 sq.deg (Udalski et al. 2015). OGLE-IV monitors regularly the Galactic Bulge, Southern Galactic Disk and 700 sq.deg. region around the Magellanic Clouds with depth of 22 mag in I-band per single image and $\sim 24$ mag on stacks.

Within OGLE we search for TDEs in both real-time datał (Wyrzykowski et al. 2014) and archival data. State-of-the-art Difference Imaging Analysis and well sampled images of galaxies allow for very precise astrometry of centres of galaxies. We have located about 6 million galaxies and conducted subtraction at their very centres, yielding light curves covering years 2010-2016. We found candidates for AGNs, supernovae and couple of longterm events, likely TDEs. Their detailed analysis is on-going. These data will be used to derive first unbiased TDE-rates. We have also identified some very short-duration nuclear flares, which could be related to low-mass black hole TDEs (Wyrzykowski et al. 2014, MacLeod, M. et al. 2016).

$\dagger$ http://ogle.astrouw.edu.pl

$\ddagger$ http://ogle.astrouw.edu.pl/ogle4/transients/ 


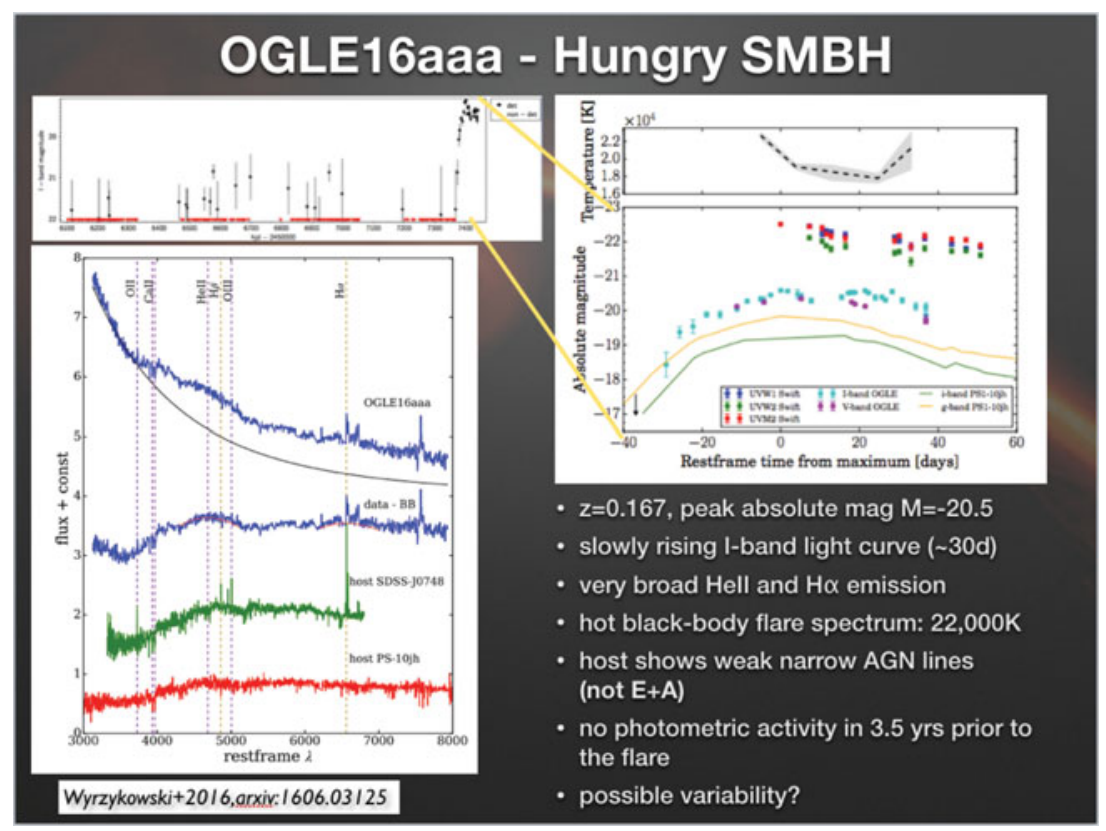

Figure 1. OGLE16aaa: first TDE candidate from OGLE (Wyrzykowski et al. 2016b)

\section{OGLE16aaa - Hungry SMBH}

OGLE16aaa, found in January 2016, was classified as OGLE's first TDE detected in real-time (Wyrzykowski et al. 2016b). Its spectral and multi-colour follow-up revealed high temperatures $(\sim 20,000 \mathrm{~K})$ remaining at that level over the duration of the event. Detection of very broad HeII and $\mathrm{H} \alpha$ emission features, along with absolute magnitude above $-20.5 \mathrm{mag}$, were consistent with a current picture of TDEs (Arcavi et al. 2014).

However, the host galaxy of OGLE16aaa did not belong to a canonical and rare E+A type of galaxies (French et al. 2016), but exhibited narrow forbidden emission lines, consistent with a weak AGN, similarly to another TDE candidate, SDSS J0748. This hints that TDEs could have been notoriously missed in previous searches, requiring a quiescent galaxy with no sign of AGN activity, leading to biases in TDE rates. The weak-AGN would also be a sign of previous episodes of stellar consumption by the SMBH, hundreds or thousands years ago.

\section{Gaia}

Gaia is the ESA's space astrometric mission in operation since mid-2014 with its main aim to derive parallaxes and proper motions of 1 billion stars of the Milky Way. It scans the entire sky with its two $1.4 \mathrm{~m}$ telescopes, therefore it allows for fairly rapid detection of transient astrophysical phenomena in the Gaia Photometric Science Alerts system $\dagger$. It reaches around $20.5 \mathrm{mag}$ in its broad G-band and a typical cadence is 30 days.

Since Gaia is an astrometric space mission, it yields very precise positional measurements. Currently, the positional accuracy reaches about 100 mas, however, it should be possible soon to improve these measurements to $100 \mu \mathrm{as}$, i.e., of order of $100 \mathrm{pc}$ in physical distance for typical observed galaxies.

Moreover, each Gaia's photometric measurement is accompanied with low-dispersion $(\mathrm{R}<100)$ red and blue spectra $(\mathrm{BP} / \mathrm{RP})$. The spectra alone can help distinguish major

$\dagger$ http://gsaweb.ast.cam.uk/alerts 


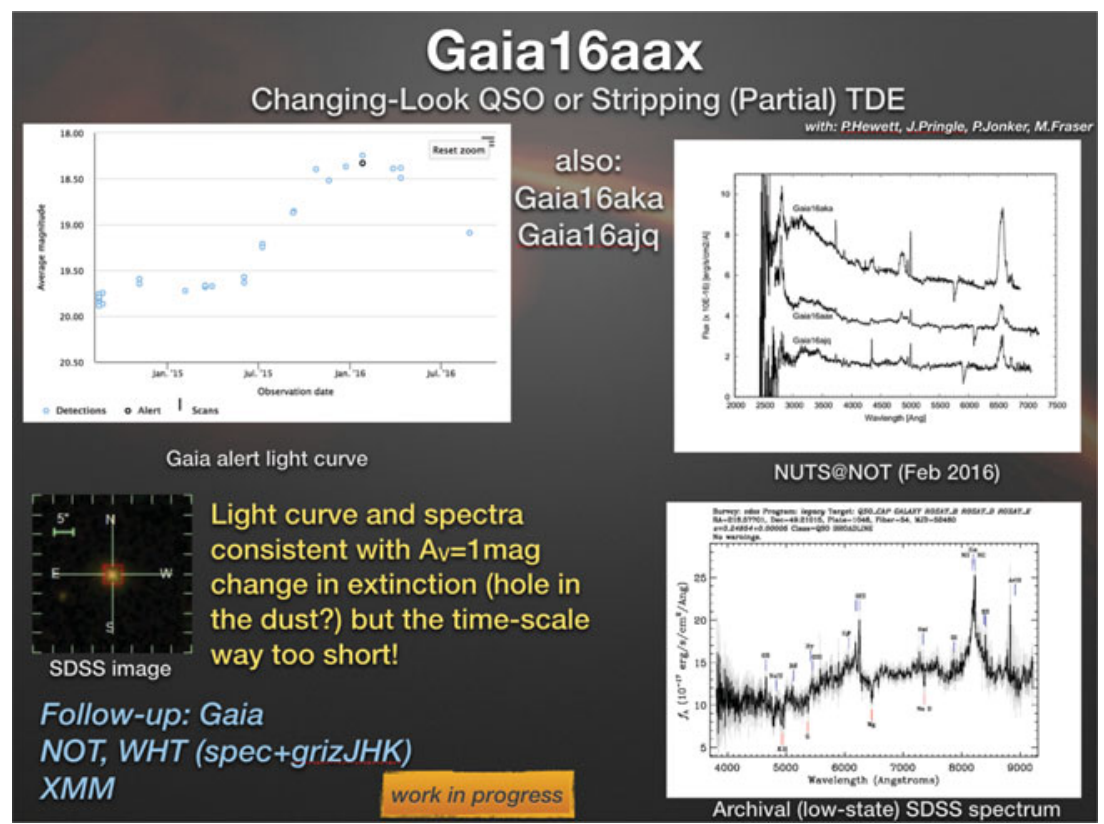

Figure 2. Gaia16aax and other nuclear flares from Gaia. Candidates for changing-look AGNs or partial TDEs.

types of transients, in particular, the most common SN Type Ia can be recognised based on their broad spectral lines. This feature of Gaia allows to immediately pick potentially interesting transients of rare type, in particular Tidal Disruption Events (TDE), which spectra typically show hot black body continuum.

Among the candidates for nuclear flares, so far Gaia has detected a handful, however, none of them were obvious cases of TDEs. However, Gaia16aax, along with Gaia16aka and Gaia16ajq, were found to be nuclear flares, and their NOT spectra showed blue continuum with broad $\mathrm{H} \alpha$ and $\mathrm{H} \beta$ and narrow AGN lines. Among possible explanations we consider a "changing-look" AGN or Stripping (Partial) TDE.

\section{Stellar mass black holes via microlensing with OGLE and Gaia}

Mass distribution of Galactic black holes is a key issue connected with our understanding of the late stages of the stellar evolution at its low-mass end and with recent detections of very massive products of black hole mergers via gravitational waves, at its heavymass end. Gravitational microlensing is probably the only channel which can increase the number of candidate black holes, both single and in binaries.

There is more than 15000 microlensing events discovered so far, however vast majority have unknown mass/distance/velocity due to degeneration of these parameters in the microlensing model. Gould (2000) predicted that $0.8 \%$ of the events are due to black holes, that means that about a dozen of events detected every year have a black hole as a lens.

In order to uniquely derive the mass and distance of the lens, and hence to conclude on its nature by comparing to the observed light, one requires to measure two observables: microlensing parallax, $\pi_{E}$ (from photometry), and angular size of the Einstein Ring, $\theta_{E}$ (from astrometry). Combination of ground-based (e.g. OGLE) photometry and Gaia astrometric time-series, would allow to measure masses and distances of nearly all microlensing events occurring on the sky, hence recognising lensing black holes. 


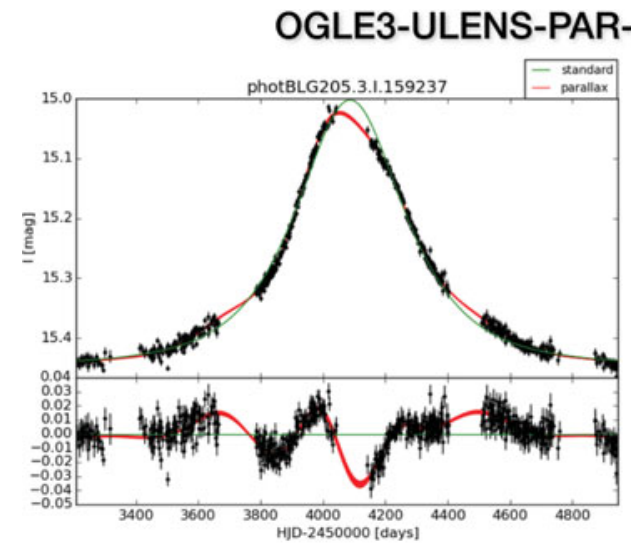

OGLE photometry (2001-2008)

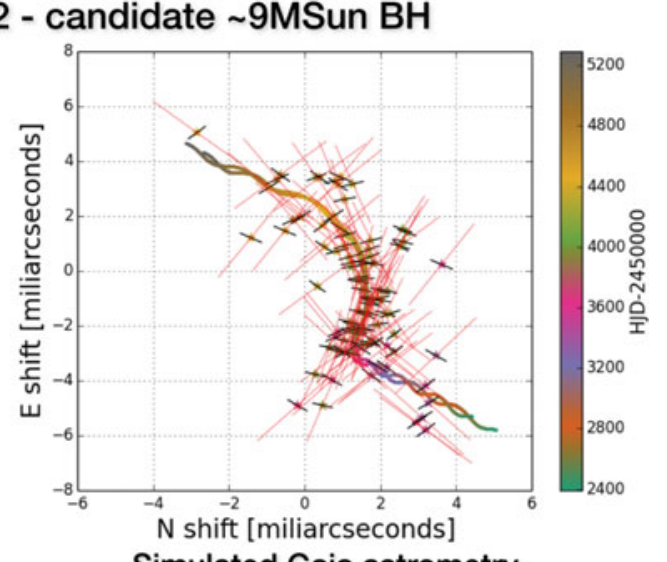

Simulated Gaia astrometry

Figure 3. Combination of ground-based photometry and Gaia's astrometry time-series will yield masses and distances of microlensing events, hence the confirmation of lensing black holes (Wyrzykowski et al. 2016a). Left panel shows an example of an old microlensing event, most likely due to a black hole. Right panel shows simulated Gaia astrometric time-series with best fit model (solid line).

OGLE3-ULENS-PAR-02, which occurred in years 2002-2008. is the best candidate for a $\sim 9 \mathrm{M}_{\odot}$ black hole lens (Wyrzykowski et al. 2016a). A similar event, discovered now during the operation of Gaia, would produce a microlensing astrometric deviation to the parallax+proper motion curve of the source, as shown in Fig.3.

\section{Summary}

- OGLE and Gaia are well suited for finding transients in galaxy centres

- Advantages: superb astrometry (Gaia, OGLE); instantaneous low-resolution spectra (Gaia)

- TDEs can be found also around active (or formerly active) black holes (OGLE16aaa) - most TDE searches so far excluded AGN hosts - is there a bias in TDE rates computation?

- Follow-up programme: spectroscopy (VLT, SALT, NOT, WHT); photometry (OPTICON Time-Domain network)

- Gaia will help find Galactic single/binary stellar mass black holes via microlensing

\section{Acknowledgments}

We thank for help at various stages of this work from numerous people, in particular Drs. Zuzanna Kostrzewa-Rutkowska, Nikolay Britavskiy, Peter Jonker, Jochen Greiner, Iair Arcavi, James Guillochon, Morgan Fraser, Nadejda Blagorodnova. We also acknowledge work of the OGLE group, PESSTO group, ESA Gaia DPAC and Gaia Photometric Science Alerts Team.

\section{References}

Arcavi, I., et al. 2014, ApJ, 793, 38

Gould, A. 2000, ApJ, 535, 928

French, K. D., Arcavi, I., \& Zabludoff, A. 2016, ApJ, 818, L21

LaMassa, S. M., et al. 2015, ApJ, 800, 144

Lawrence, A., et al. 2016, MNRAS, 463, 296 
MacLeod, C. L., et al. 2016, MNRAS, 457, 389

MacLeod, M, et al. 2016, ApJ, 819, 3

Ruan, J. J., et al. 2016, ApJ, 826, 188

Shappee, B. J., et al. 2014, ApJ, 788, 48

Udalski, A., Szymański, M. K., \& Szymański, G. 2015, AcA, 65, 1

Wyrzykowski, Ł., et al. 2014, AcA, 64, 197

Wyrzykowski, Ł., et al. 2016a, MNRAS, 458, 3012

Wyrzykowski, Ł., et al. 2016b, arXiv:1606.03125v2, accepted for MNRAS Letters 